\title{
Paper-based DPPH Assay for Antioxidant Activity Analysis
}

\author{
Kitima Sirivibulkovit,* Souksanh Nouanthavong, ${ }^{*} * *$ and Yupaporn Sameenor ${ }^{* \dagger}$ \\ *Department of Chemistry and Center of Excellence for Innovation in Chemistry, Faculty of Science, \\ Burapha University, Chon Buri, 20131, Thailand \\ **Savannakhet Teacher Training College, Savannakhet, Lao PDR
}

\begin{abstract}
We report on a paper-based 2,2-diphenyl-1-(2,4,6-trinitrophenyl)hydrazyl (DPPH) assay for a simple, inexpensive, low reagent and sample consumption and high throughput analysis of antioxidant activity. The paper-based device was fabricated using a lamination method to create a $5-\mathrm{mm}$ in diameter circular test zone that was embedded with a DPPH reagent. The analysis was carried out in one-step by dropping an antioxidant/sample onto the test zone. After reduction by the antioxidant, the DPPH radicals become stable DPPH molecules, resulting in a change in color from deep violet to pale yellow. The violet color intensity of DPPH was inversely proportional to the antioxidant activity of the samples, and was measured using imaging software. A high precision and a low limit of detection were found in the analysis of six standard antioxidants including gallic acid, trolox, ascorbic acid, caffeic acid, vanilliic acid and quercetin. The device was then validated against the traditional spectrophotometric DPPH assay by analyzing the antioxidant activity of 7 tea samples. The results showed no significant difference for gallic acid equivalent for all 7 samples obtained from the two methods at the $95 \%$ confidence level, indicating that the developed method was reliable for antioxidant activity analysis of real samples. Finally, the paper-based DPPH device was found to be stable over 10 days when stored in a refrigerator $\left(2-4^{\circ} \mathrm{C}\right)$, making it an easy-to-use device for end-users.
\end{abstract}

Keywords DPPH assay, paper-based devices, antioxidant activity

(Received January 12, 2018; Accepted February 16, 2018; Published July 10, 2018)

\section{Introduction}

Antioxidants are molecules that can interact and inhibit the initiation or propagation of oxidizing chain reactions generated by reactive free radicals before vital molecules are damaged. ${ }^{1}$ They cause many diseases including cancer, Parkinson's disease, Alzheimer's disease, ${ }^{2}$ cardiovascular disorders and neurological diseases. ${ }^{3}$ Antioxidants can inhibit free-radical reactivity through several mechanisms including the donation of hydrogen, radical scavenging and singlet oxygen quenching. ${ }^{4}$ Antioxidants occur naturally and through synthetic chemical processes. Examples of natural antioxidants are vitamin E, $\mathrm{C}$ and $\beta$-carotene ${ }^{5}$ and those of synthetic antioxidants are tertbutylhydroquinone (E-319), butylated hydroxyl anisole (BHA; E320) butylated hydroxytoluene (BHT; E321) and propyl gallate (E-311). ${ }^{6}$

Current methods for determining antioxidant activity are based on spectrophotometric determination through hydrogen atom transfer (HAT) and single electron transfer (SET) mechanisms. ${ }^{7} \quad$ These assays included 2,2'-azino-bis(3ethylbenzothiazoline-6-sulfonic acid (ABTS) assay, ${ }^{8}$ 2,2-diphenyl-1-(2,4,6-trinitrophenyl)hydrazyl (DPPH) radical scavenging activity, ${ }^{9}$ oxygen radical absorbance capacity (ORAC) assay, ${ }^{10}$ ferric reducing antioxidant potential (FRAP) assay $^{11}$ and cupric reducing antioxidant capacity (CUPRAC)

K. S. and S. N. contributed equally to this work.

† To whom correspondence should be addressed.

E-mail: yupaporn@buu.ac.th assay. ${ }^{12}$ Among these assays, the DPPH assay is simple and one of the most widely used methods. ${ }^{13}$ It is based on reduction of the violet DPPH radical by the antioxidant via a hydrogen atom transfer mechanism to cause a change in the color to stable paleyellow DPPH molecules. The remaining violet DPPH radical is measured by a UV-Vis spectrophotometer at approximately $515-520 \mathrm{~nm}$ to determine the antioxidant activity, as shown in Fig. 1. 14-16 This test provides useful information on the antioxidant capacity to donate hydrogen atoms, on the reaction's reducing capacity, and on mechanism between the free radical and the antioxidant. The test is also simple, since it is associated with only the DPPH radical reagent and the antioxidant. The assay also requires mild experimental conditions over other traditional antioxidant assays that require additional treatments of sample and reagents, such as high temperatures and or oxygen supply. ${ }^{17}$ The traditional DPPH assay, however, suffers from a requirement for a large volume of freshly prepared DPPH solution, normally $2-4 \mathrm{ml}$ per sample. ${ }^{18,19}$ Storage of the DPPH stock solution at low temperatures was recommended, ${ }^{20}$ but later found to be unstable. ${ }^{21}$ To overcome these limitations, DPPH sensing film was used for rapid and low-cost screening of antioxidant activity, but was only used for qualitative measurements. ${ }^{22}$ More recently, a DPPH dry reagent 96 well plate array was developed to provide for a quantitative analysis of antioxidant capacity. ${ }^{13}$ Although, the assay was fast and simple, a relatively expensive microplate spectrophotometer was required for detection.

Microfluidic paper-based analytical devices ( $\mu$ PADs) have gained attention and have been successfully used as a detection platform for several applications, including environmental 


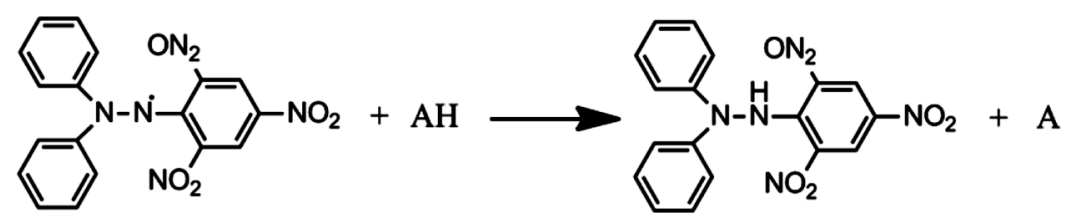

Fig. 1 Reaction of antioxidant and DPPH free radical. AH is an antioxidant donor molecule, and A is a free radical produced.

monitoring, health diagnosis and food safety. ${ }^{23-25}$ The $\mu$ PADs can be simply made from inexpensive chromatography or filter paper. They are fabricated by forming hydrophobic barriers in hydrophilic paper producing hydrophilic channels for liquid flow. ${ }^{26}$ A number of fabrication techniques have been developed for $\mu$ PADs including lamination, ${ }^{27}$ wax printing, ${ }^{28}$ inkjet printing, ${ }^{29}$ photolithography ${ }^{30,31}$ and flexographic printing. ${ }^{32}$ The advantages of using $\mu$ PADs over traditional detection platforms are that the paper is widely available at a low cost. The device can be designed to be easy to use so that it can be delivered to end-users. The analysis requires only microliter volumes of the sample and reagents. The device is thin, light and small making it portable for use on-site. . $^{33,34}$

Previously, $\mu$ PADs were applied for antioxidant activity analysis so as to allow for simple, fast and inexpensive tests. Nanoparticles were employed for antioxidant activity analysis on the $\mu \mathrm{PAD}$ to determine the reducing power of an antioxidant toward metal nanoparticles. ${ }^{35-37}$ The capacity of antioxidant to reduce $\mathrm{Au}^{3+}$ to form gold nanoparticles was measured colorimetrically on paper. ${ }^{37}$ The reducing power of the antioxidant was also determined by the nanoceria on the $\mu \mathrm{PAD}$, where $\mathrm{Ce}^{4+}$ on the nanoceria surface was reduced to $\mathrm{Ce}^{3+}$ by the antioxidant, leading to a change in color from yellow to brown. ${ }^{35,36}$ The radical scavenging activity of the antioxidant has also been measured using the DPPH assay on $\mu$ PADs. However, only two antioxidant standards, including gallic acid ${ }^{38}$ and butylated hydroxytoluene ${ }^{39}$ have been investigated. No further investigation on the optimization of the assay as well as the stability of the developed devices has been carried out. ${ }^{38,39}$ Moreover, the calibration curve for DPPH assay was generated using $\mu$ PADs that have different features to those used for sample analysis, which could potentially affect the method accuracy. ${ }^{39}$

Here, we present a fully investigated study of a paper-based DPPH assay for low cost, simple to operate, rapid, low reagent consumption and high throughput analysis of antioxidant activity. The $\mu$ PAD was fabricated by the lamination method, and the DPPH reagent was deposited on the detection zone of the device, and allowed to dry. For antioxidant analysis, the sample was simply added into the detection zone to react with the pre-deposited DPPH. The decreasing of the deep violet DPPH radical color intensity was quantified using imaging software. Here, we first studied the optimal concentration of DPPH radicals deposited on the paper and the reaction time to obtain a sensitive response of the antioxidant analysis. We next studied the analysis of several standard antioxidants to determine the performance of the developed paper-based DPPH assay in its ability to determine various types of antioxidants. The performance of the paper-based DPPH assay was further validated against the traditional DPPH assay by analyzing the antioxidant activity of seven types of tea samples to determine the accuracy. The results showed no significant difference at the 95\% confidence level of the antioxidant activity of all samples obtained from the two methods. Finally, the paper-based DPPH

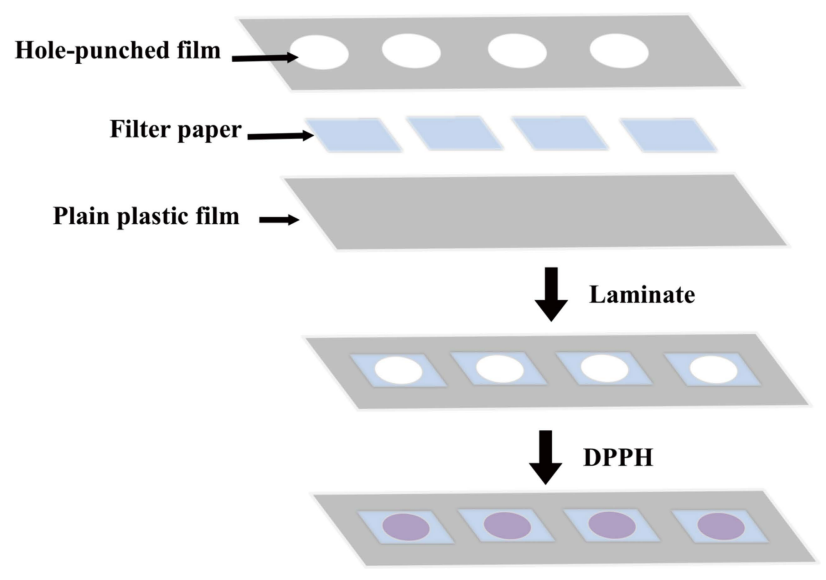

Fig. 2 Paper-based DPPH device fabrication using the lamination method. Circular holes were cut on the lamination film (top layer). Whatman filter paper No. 4 was cut into small pieces $(1 \times 1 \mathrm{~cm})$ (middle layer) and arranged under the hole of the plastic layer. Plain lamination film was put at the bottom layer. The three layers were laminated to create the paper-based devices. The DPPH was finally deposited on the detection zone and allowed to dry.

devices were found to be stable when stored in a refrigerator for at least 10 days.

\section{Experimental}

\section{Reagents and equipment}

Whatman No. 4 filter paper was purchased from Whatman International Ltd, China. Laminate film 100 micron $(216 \times$ $303 \mathrm{~mm}$ ) was purchased from Neocal, Thailand. Laminator (PapermonsterLA4, 397) was obtained from Matin Yale International, Germany. The antioxidant standards including 6-hydroxy-2,5,7,8-tetra-methylchroman-2-carboxylic acid (trolox), 3,4,5-trihydroxylbenzoic acid (gallic acid), 4-hydroxy3-methoxybenzoic acid (vanillic acid), 2-oxo-L-threo-hexono (L-ascorbic acid), 3-(3,4-dihydroxyphenyl)-2-propenoic acid (caffeic acid) and 2-(3,4-dihydroxyphenyl)-3,5,7-trihydroxy-4H-chromen-4-one (Quercetin) as well as the reagents including 2,2-diphenyl-1-picrylhydrazyl (DPPH) and methanol were from Sigma-Aldrich. Tea samples were obtained from a local supermarket in Chon Buri province, Thailand. Deionized water (DI) was prepared using a BarnsteadTM e-pureTM ultrapure water purification system and was used throughout the experiments.

All stock solutions of antioxidant standards were prepared in methanol and working solutions were prepared daily by dilution of the stock solution using deionized water. Tea samples were prepared by heating $2 \mathrm{~g}$ tea in $200 \mathrm{~mL}$ of DI water at $80^{\circ} \mathrm{C}$ for 5 min. After cooling to room temperature, the tea solution was 


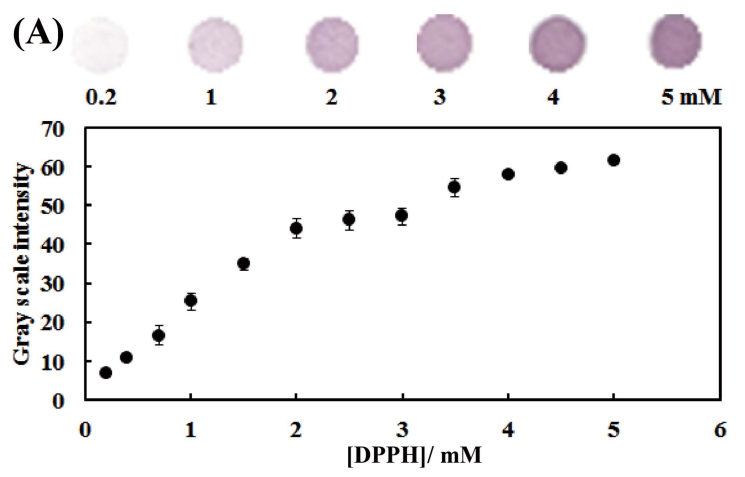

(B)

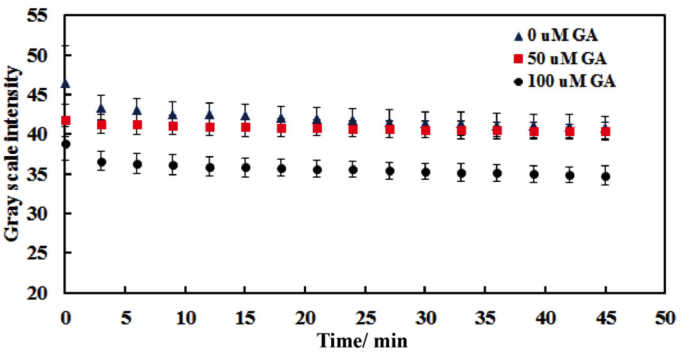

Fig. 3 Optimization of paper-based DPPH assay (A) color response as a function of the DPPH concentration $(n=3)$, (B) color response of the paper-based DPPH assay with gallic acid as an antioxidant standard to the reaction time $(n=3)$.

filtered through Whatman number 1 filter paper and the sample was analyzed concerning its antioxidant activity.

\section{Paper-based DPPH device fabrication}

The paper-based device was fabricated using a lamination method (Fig. 2). ${ }^{40}$ The device consisted of three layers. The top layer was a plastic lamination film that was punched to have circular holes of $0.5 \mathrm{~cm}$ in diameter. The middle layer consisted of a Whatman No. 4 filter paper with a size of $1 \times 1 \mathrm{~cm}$. The bottom layer consisted of plain plastic lamination film. The three layers were laminated to produce a portable paper-based device with a $0.5-\mathrm{cm}$ in diameter circular detection zones. To create a paper-based DPPH device, $1.0 \mu \mathrm{L}$ of $2 \mathrm{mM} \mathrm{DPPH}$ solution was added to the detection zone. After drying, the device was ready to use for the antioxidant activity analysis, and was stable for at least one month when stored at $2-4^{\circ} \mathrm{C}$.

\section{Analysis of antioxidants activity using a paper-based DPPH} assay

The analysis of antioxidant activity using the device described above can be performed in only one step by simply adding $1.0 \mu \mathrm{L}$ of the standard antioxidant solution or sample to the detection zone. The reaction was allowed to proceed for $30 \mathrm{~min}$ in the dark, which was sufficient time for the detection zone to dry. The picture of the devices was captured using a scanner (CanoScanLiDE 110, Cannon, Vietnam) and the intensity of DPPH that was inversely proportional to the antioxidant activity was measured using imageJ software (NIH, USA). In this work, various antioxidant standards were measured using the developed paper-based DPPH assay including gallic acid, trolox, ascorbic acid, caffeic acid, vanilliic acid and quercetin. The analytical features including linear range, reproducibility and limit of detection were investigated from the analysis of these standards. Gallic acid was used as a standard antioxidant in measurements of the sample antioxidant activity that were reported as gallic acid equivalent (GAE) in units of $\mu \mathrm{molGA} / \mathrm{g}$ sample. The antioxidant activity of the samples obtained from the developed method was compared to those obtained using the traditional DPPH method in order to determine the accuracy of the developed paper-based DPPH assay.

\section{DPPH assay optimization on the paper-based devices}

The initial concentration of the DPPH solution as well as the reaction time were studied in order to optimize the condition for the paper-based devices. For the initial concentration optimization, the experiment was performed without the addition of antioxidants. DPPH solutions in the concentration range $0.1-5.0 \mathrm{mM}$ were prepared in methanol and $1.0 \mu \mathrm{L}$ of each concentration added to the detection zones $(n=5)$. The zones were allowed to dry and the violet color intensity was measured as described above.

Optimization of the reaction time was carried out by adding $1.0 \mu \mathrm{L}$ of $2 \mathrm{mM}$ DPPH on the paper-based devices, and allowed to dry, followed by $1.0 \mu \mathrm{L}$ gallic acid or caffeic acid solution. Assay mixtures were allowed to react for 3 to $39 \mathrm{~min}$ and analyzed as described.

\section{Stability study}

The stability of the paper-based DPPH devices was determined by depositing $1.0 \mu \mathrm{L}$ of $2 \mathrm{mM}$ DPPH on the detection zones and then wrapping them in aluminium foil to protect from light. The devices were stored in a refrigerator $\left(2-4^{\circ} \mathrm{C}\right)$ and at room temperature $\left(26-31^{\circ} \mathrm{C}\right)$ over 30 days. Gallic acid $(1.0 \mu \mathrm{L}$ of 0 and $250 \mathrm{mM}$ ) was used for the test. Each experiment was performed in triplicate.

\section{Results and Discussion}

\section{Assay optimization}

In the DPPH assay, the antioxidant activity analysis is based on the inhibition of the DPPH radical by the antioxidants. The optimal initial concentration of DPPH was evaluated first to determine the assay sensitivity. The violet color intensity increased rapidly with the DPPH concentration from $0-2 \mathrm{mM}$ (Fig. 3A). When the DPPH concentration was higher than $2 \mathrm{mM}$, however, the color intensity only slightly increased, and had almost become saturated at $4 \mathrm{mM}$. Therefore, $2 \mathrm{mM} \mathrm{DPPH}$ was considered to be the optimal value to allow for a sensitiveresponse analysis of antioxidants. This result also demonstrated that we could measure antioxidant activity that scavenged DPPH radicals in a dose-response manner.

The reaction time of DPPH and antioxidant standards on the analytical paper-based device was also examined over the range of 3-39 min. Both high- and low-antioxidant activity compounds including gallic acid and caffeic acid, respectively, were employed for this study. For the gallic acid standard, the color intensity of DPPH decreased quickly upon the addition of gallic acid for about $3 \mathrm{~min}$, and then gradually decreased with a near-zero slope of the reaction time curve (Fig. 3B). For caffeic acid, on the other hand, the reaction required more than $21 \mathrm{~min}$ to complete, as can be seen in the decreasing of slope, and 
became steady after that (Fig. S1). Therefore, a reaction time of $30 \mathrm{~min}$ was selected and used throughout this work to ensure that the reaction was complete for both high- and low-antioxidant activity compounds. Although the selected reaction time of the developed method is similar to that of the traditional method, multiple samples can be analyzed at the same time, and thus increasing the throuhput.

\section{Analytical performance}

Under the optimal conditions, the paper-based DPPH device was used to analyze six antioxidant standards including gallic acid, trolox, ascorbic acid, caffeic acid, vanilliic acid and quercetin to study the ability of the method to analyze several types of antioxidants. The analytical features from the analysis of all antioxidant standards including linearity, reproducibility and detection limit were demonstrated, and are summarized in Table 1. The paper-based DPPH assay responded well to all of the antioxidant standard investigated. The color intensity is inversely proportional to antioxidant concentrations (Fig. 4) The linear range from the analysis of each antioxidant standard is shown in the insets. The reproducibility of the assays was determined by performing 10 replicate analyses of antioxidan standards at three different concentrations in the linear range, and reported as the relative standard deviation (\%RSD). A high reproducibility of the method was obtained with the \%RSD being in the range of $1.5-17 \%$ for all antioxidant standards investigated.

The limit of detection (LOD) from each antioxidant analysis, defined as the antioxidant concentration causing a decrease in DPPH signal three times the signal-to-noise ratio relative to the

Table 1 Summary of the analytical performance of antioxidant analysis using a paper-based DPPH assay

\begin{tabular}{llllll}
\hline Antioxidant & Sensitivity & Linear range & LOD & $R^{2}$ & $\begin{array}{c}\text { \%RD } \\
(n=10)\end{array}$ \\
\hline Gallic acid & $-0.072 \mu \mathrm{M}^{-1}$ & $0-500 \mu \mathrm{M}$ & $37.9 \mu \mathrm{M}$ & 0.991 & $6.7-16$ \\
Trolox & $-0.034 \mu \mathrm{M}^{-1}$ & $200-1400 \mu \mathrm{M}$ & $295 \mu \mathrm{M}$ & 0.991 & $2.6-12$ \\
Ascorbic acid $-0.029 \mu \mathrm{M}^{-1}$ & $0-1100 \mu \mathrm{M}$ & $300 \mu \mathrm{M}$ & 0.987 & $2.6-7.3$ \\
Caffeic acid & $-0.052 \mu \mathrm{M}^{-1}$ & $300-700 \mu \mathrm{M}$ & $300 \mu \mathrm{M}$ & 0.990 & $4.7-7.9$ \\
Vanillic acid & $-0.534 \mathrm{mM}^{-1}$ & $0-50 \mathrm{mM}$ & $10 \mathrm{mM}$ & 0.982 & $1.5-17$ \\
Quercetin & $-0.061 \mu \mathrm{M}^{-1}$ & $100-600 \mu \mathrm{M}$ & $97.8 \mu \mathrm{M}$ & 0.996 & $2.2-17$ \\
\hline
\end{tabular}

A

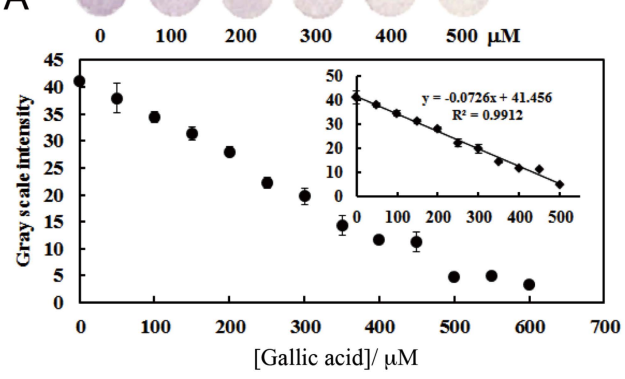

C

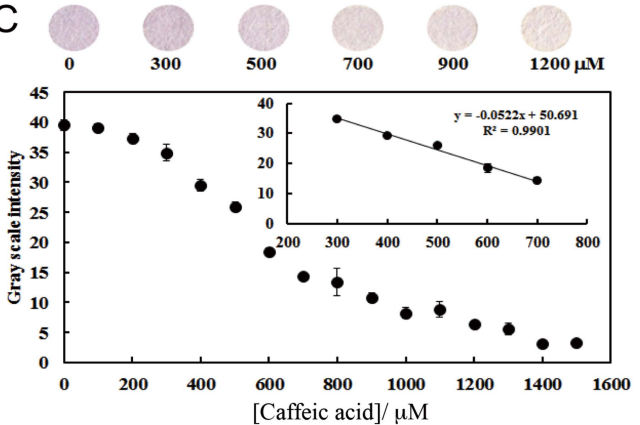

$E$

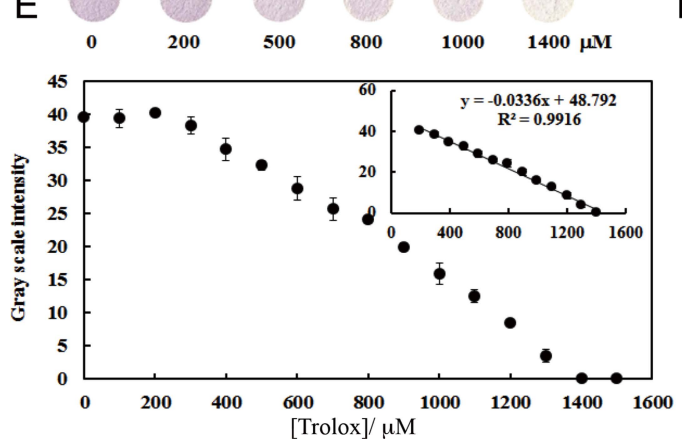

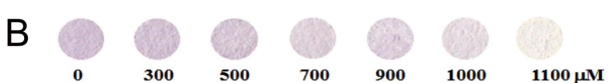

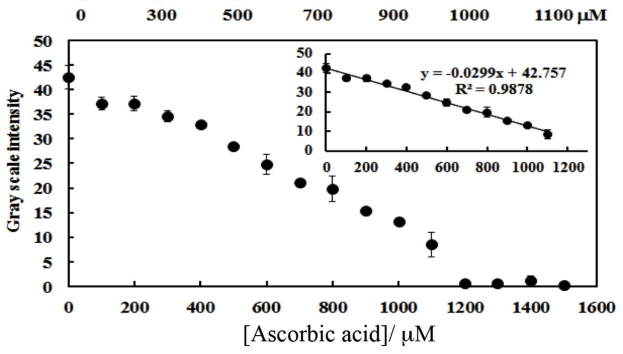

D

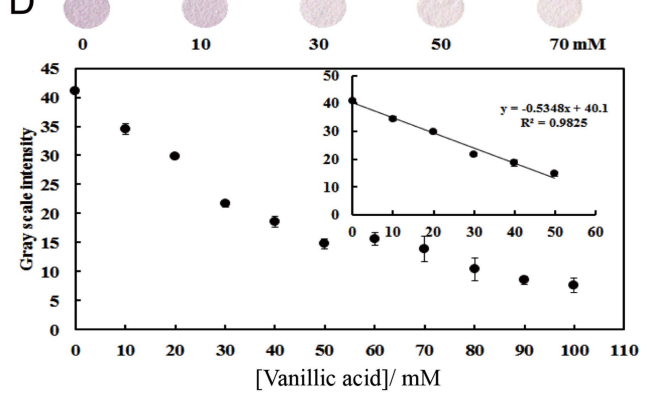

$\mathrm{F}$
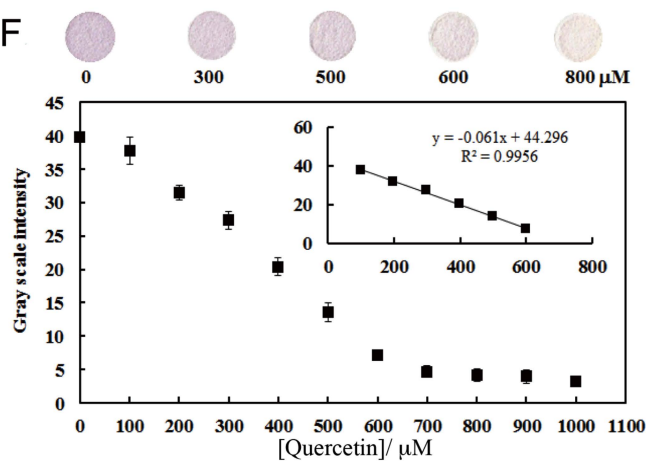

Fig. 4 Dose-response curves for the detection of antioxidant standards using the paper-based DPPH assays (A) gallic acid, (B) ascorbic acid, (C) caffeic acid, (D) vanillic acid, (E) trolox, and (F) quercetin. Photographs of the actual responses are shown across the top of each plot. The inset of each plot is the linear range of calibration curve $(n=3)$. 


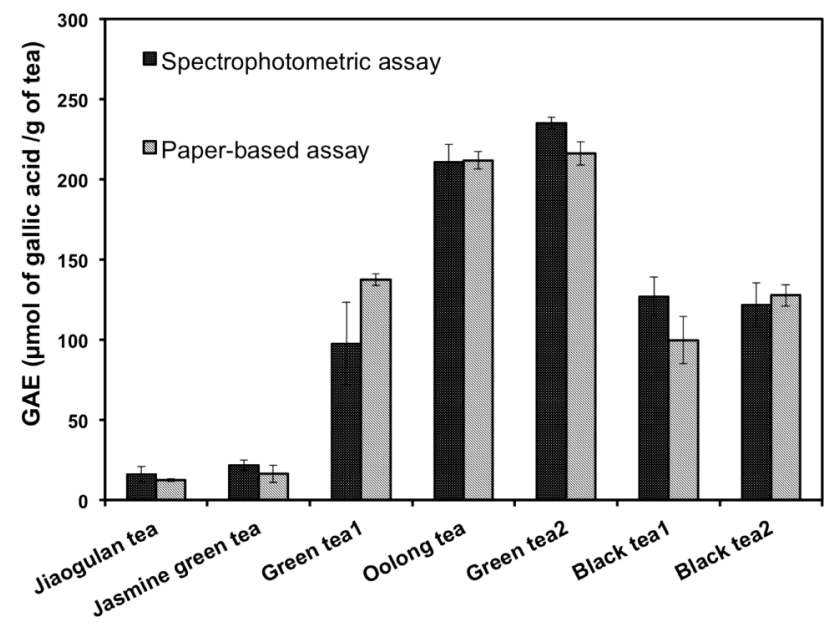

Fig. 5 Antioxidant activity expressed as GAE in $\mu$ mol gallic acid equivalent/g tea unit from the analysis of seven tea samples using the paper-based DPPH assay and the traditional spectrophotometric DPPH assay $(n=3)$.

control, was found to depend on the reducing capacity of the antioxidant. ${ }^{41}$ The chemical structures and the functional groups that play key roles in antioxidant activity of evaluated standards are given in the supporting information (Table S1). The dotted circle demonstrates the position of the $\mathrm{OH}$ group on the phenolic ring that can be oxidized by the DPPH radicals. These positions affected the antioxidant activity of the standard antioxidants. The higher is the number of hydroxyl groups on the phenolic ring that provides $\mathrm{H}$-donating transfer to the DPPH free radical to form the $o$-quinone, ${ }^{19,42}$ the higher is the reducing capacity and lower LOD. The overall trend of the LODs from the standard antioxidant analysis can be arranged in the order gallic acid $>$ quercetin $>$ trolox $>$ ascorbic acid $=$ caffeic acid $>$ vanillic acid which was similar to previous reports. ${ }^{43,44}$ Gallic acid has three $-\mathrm{OH}$ groups for reducing $\mathrm{DPPH}^{*}$ and can form $o$-quinone at 3 positions. Quercetin has four -OH groups, but only 2 can form $o$-quinone, ${ }^{45}$ resulting in a higher LOD. Both ascorbic acid and caffeic acid have two -OH groups in the molecules resulting in higher LOD than gallic acid and quercetin. Ascorbic acid has an enolic form that is easily oxidized, ${ }^{46}$ whereas caffeic acid has two -OH groups which form o-quinone. Trolox and vanillic acid have one-OH group available on the phenolic ring, but trolox is a lipophilic antioxidant that can react well with the DPPH free radical in alcoholic solvents, ${ }^{47}$ resulting in a lower LOD than that of ascorbic acid, caffeic acid and valinilic acid. In comparison to the previous work, the device gave a lower LOD for analysis of caffeic acid. ${ }^{48}$

\section{Analysis of antioxidants activity in tea samples}

The paper-based DPPH assay developed in this work was validated against the traditional DPPH assay using seven tea extracts from different types of tea including Jiaogulan tea, Jasmine tea, Oolong tea, green tea and black tea. The antioxidant activity expressed as GAE was measured using both methods and compared (Fig. 5). There was no significant difference in GAE between the two methods based on a $95 \%$ confidence interval (two tailed $P=0.8114$ ). The high degree of equivalence between the two methods indicates that the paper-based DPPH assay is appropriate to analyze the antioxidant activity in real samples. More importantly, however, the paper-based DPPH assay required approximately 50-times less sample mass and 100 -times less reagent volume than in traditional DPPH assay.

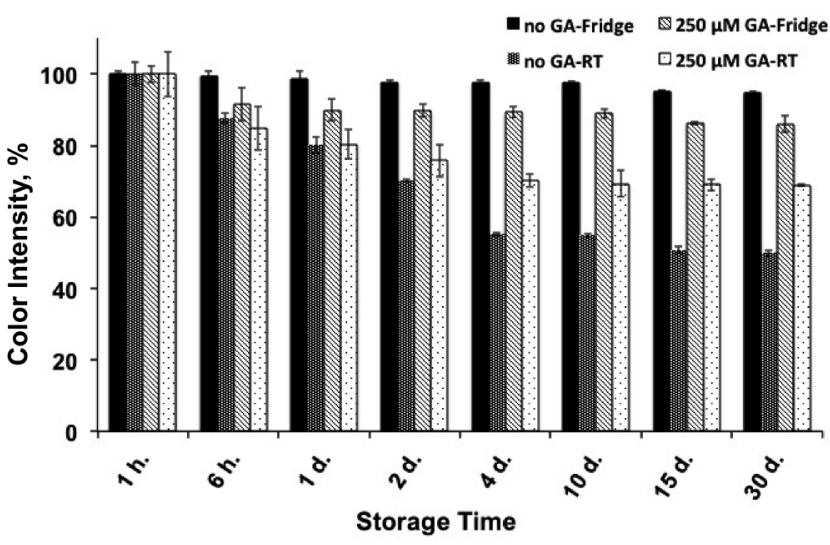

Fig. 6 Storage stability of the paper-based DPPH assay in the refrigerator $\left(2-4^{\circ} \mathrm{C}\right)$ and at room temperature $\left(26-31^{\circ} \mathrm{C}\right)$ demonstrated as \%decrease in color with $100 \%$ being the response at day 0 for the analysis of 0 and $250 \mu \mathrm{M}$ gallic acid (GA).

These improvements enable further applications to be possible for the analysis of antioxidant activity in the natural product extract collection of samples that have low mass in each fraction. The assay also allowed for high throughput analysis where more than 20 samples can be analyzed simultaneously by simply adding a drop of samples onto the developed devices.

\section{Assay stability}

The stability of the paper-based DPPH assay was investigated by storing the devices at two different temperatures for different times. A plot of the $\%$ color intensity, defined as \%decrease with $100 \%$ being the response of the freshly prepared devices, as a function of the storage time for the analysis of 0 and $250 \mu \mathrm{M}$ gallic acid (GA) is shown in Fig. 6. The devices that were stored in the dark at room temperature showed a significant loss of stability after 1 day. The paper-based devices that were stored in a refrigerator, on the other hand, were found to be relatively stable for 10 days. The results observed here were similar to a previous report, which indicated that DPPH was easily decomposed at room temperature, ${ }^{45}$ but more stable at low temperature. ${ }^{13}$ Another important factor that may affect the stability of our device is humidity in tropical regions such as Thailand. High humidity can cause a degradation of the reagent in the test strip, such as glucose. ${ }^{49}$ We anticipated that the DPPH paper-based devices kept at a lower humidity condition would be more stable for a longer period of time.

\section{Conclusions}

A paper-based DPPH assay has been developed for the analysis of antioxidant activity by using colorimetric detection with a desktop scanner and imaging software. The assay provides for fast, simple, low sample and reagent consumption, low cost and high-throughput analysis of antioxidant activity. The devices can effectively analyze different types of antioxidants with high reproducibility and low limits of detection. The device required approximately 100-times less sample and reagent volume than the traditional DPPH assay, and as low as a two-microliter total volume of the reagent and the sample was required. The accuracy of the method was comparable to the traditional DPPH assay at the $95 \%$ confidence interval based on the analysis of seven different types of tea samples. The device was found to be stable for more than 10 days when stored at $2-4^{\circ} \mathrm{C}$. 
This result indicated that the paper-based DPPH assay could be further developed for a commercially ready-to-use platform where the user needs only adding a single drop of samples to quantitatively measure antioxidant activity.

\section{Acknowledgements}

This work was financially supported by the Research Grant of Burapha University through National Research Council of Thailand (Grant No. 72/2558 and 93/2559) and the Center of Excellence for Innovation in Chemistry (PERCH-CIC), Commission on Higher Education, Ministry of Education. We would also like to thank Prof. Dr. Federick W. H. Beamish and Prof. Dr. Ron Beckett, Faculty of Science, Burapha University for their comments and correction.

\section{Supporting Information}

This material is available free of charge on the Web at http:// www.jsac.or.jp/analsci/.

\section{References}

1. A. Ismail, Z. M. Marjan, and C. W. Foong, Food Chem., 2004, 87, 581.

2. L. A. Pham-Huy, H. He, and C. Pham-Huy, Int. J. Biomed. Sci., 2008, 4, 89.

3. R. Komeri, F. G. Thankam, and J. Muthu, Mater. Sci. Eng. $C$, 2017, 71, 100 .

4. M. Antolovich, P. D. Prenzler, E. Patsalides, S. McDonald, and K. Robards, Analyst, 2002, 127, 183.

5. A. Augustyniak, G. Bartosz, A. Čipak, G. Duburs, L. U. Horáková, W. Łuczaj, M. Majekova, A. D. Odysseos, L. Rackova, and E. Skrzydlewska, Free Radical Res., 2010, 44,1216

6. A. Wollinger, E. Perrin, J. Chahboun, V. Jeannot, D. Touraud, and W. Kunz, Comptes Rendus Chimie, 2016, 19, 754.

7. B. D. Craft, A. L. Kerrihard, R. Amarowicz, and R. B. Pegg, Comprehensive Reviews in Food Science and Food Safety, 2012, 11, 148.

8. S.-M. Jia, X.-F. Liu, D.-M. Kong, and H.-X. Shen, Biosens. Bioelectron., 2012, 35, 407.

9. V. Pedan, N. Fischer, and S. Rohn, Food Res. Int., 2016, 89, 890.

10. A. C. Kurilich, E. H. Jeffery, J. A. Juvik, M. A. Wallig, and B. P. Klein, J. Agric. Food. Chem., 2002, 50, 5053.

11. A. T. Hukkanen, S. S. Pölönen, S. O. Kärenlampi, and H. I. Kokko, J. Agric. Food. Chem., 2006, 54, 112.

12. M. Bener, M. Özyürek, K. Güçlü, and R. Apak, Anal. Chem., 2010, 82, 4252.

13. K. H. Musa, A. Abdullah, B. Kuswandi, and M. A. Hidayat, Food Chem., 2013, 141, 4102.

14. E. J. Garcia, T. L. C. Oldoni, S. M. d. Alencar, A. Reis, A. D. Loguercio, and R. H. M. Grande, Braz. Dent. J., 2012, 23, 22.

15. Z. Cheng, J. Moore, and L. Yu, J. Agric. Food Chem., 2006, $54,7429$.

16. A. Wei and T. Shibamoto, J. Agric. Food Chem., 2010, 58, 7218.

17. I. I. Koleva, T. A. Van Beek, J. P. Linssen, A. d. Groot, and L. N. Evstatieva, Phytochem. Anal., 2002, 13, 8.

18. M. R. Maria do Socorro, J. Pérez-Jiménez, S. Arranz, R. E. Alves, E. S. de Brito, M. S. Oliveira, and F. Saura-Calixto,
Food Res. Int., 2011, 44, 2100.

19. D. Villano, M. Fernández-Pachón, M. Moyá, A. Troncoso, and M. García-Parrilla, Talanta, 2007, 71, 230.

20. T. E. Shian, A. Abdullah, K. H. Musa, M. Y. Maskat, and M. A. Ghani, Sains Malaysiana, 2012, 41, 319.

21. J. Deng, W. Cheng, and G. Yang, Food Chem., 2011, 125, 1430.

22. I. M. Steinberg and S. Milardović, Talanta, 2007, 71, 1782.

23. D. M. Cate, J. A. Adkins, J. Mettakoonpitak, and C. S. Henry, Anal. Chem., 2014, 87, 19.

24. J. Hu, S. Wang, L. Wang, F. Li, B. Pingguan-Murphy, T. J. Lu, and F. Xu, Biosens. Bioelectron., 2014, 54, 585.

25. P. Jarujamrus, R. Meelapsom, S. Pencharee, A. Obma, M. Amatatongchai, N. Ditcharoen, S. Chairam, and S. Tamuang, Anal. Sci., 2018, 34, 75.

26. Y. Sameenoi, P. Panymeesamer, N. Supalakorn, K. Koehler, O. Chailapakul, C. S. Henry, and J. Volckens, Environ. Sci. Technol., 2012, 47, 932.

27. R. A. de Oliveira, F. Camargo, N. C. Pesquero, and R. C. Faria, Anal. Chim. Acta, 2017, 957, 40.

28. E. Carrilho, A. W. Martinez, and G. M. Whitesides, Anal. Chem., 2009, 81, 7091.

29. K. Abe, K. Suzuki, and D. Citterio, Anal. Chem., 2008, 80, 6928.

30. A. W. Martinez, S. T. Phillips, E. Carrilho, S. W. Thomas III, H. Sindi, and G. M. Whitesides, Anal. Chem., 2008, 80, 3699.

31. H. Asano and Y. Shiraishi, Anal. Sci., 2018, 34, 71.

32. J. Olkkonen, K. Lehtinen, and T. Erho, Anal. Chem., 2010, 82, 10246.

33. Y. Xia, J. Si and Z. Li, Biosens. Bioelectron., 2016, 77, 774.

34. P. Worramongkona, K. Seeda, P. Phansomboon, N. Ratnarathorn, O. Chailapakul, and W. Dungchai, Anal. Sci., 2018, 34, 103.

35. T. Piyanan, A. Athipornchai, C. S. Henry, and Y. Sameenoi, Anal. Sci., 2018, 34, 97.

36. E. Sharpe, T. Frasco, D. Andreescu, and S. Andreescu, Analyst, 2013, 138, 249.

37. T. G. Choleva, F. A. Kappi, D. L. Giokas, and A. G. Vlessidis, Anal. Chim. Acta, 2015, 860, 61.

38. Y. Sameenoi, P. N. Nongkai, S. Nouanthavong, C. S. Henry, and D. Nacapricha, Analyst, 2014, 139, 6580.

39. N. Nuchtavorn and M. Macka, Anal. Chim. Acta, 2016, 919, 70

40. E. M. Fenton, M. R. Mascarenas, G. P. López, and S. S. Sibbett, ACS Appl. Mater. Interfaces, 2008, 1, 124.

41. J. Xie and K. Schaich, J. Agric. Food Chem., 2014, 62, 4251.

42. C. Grajeda-Iglesias, E. Salas, N. Barouh, B. Baréa, A. Panya, and M. C. Figueroa-Espinoza, Food Chem., 2016, 194, 749.

43. W. Brand-Williams, M.-E. Cuvelier, and C. Berset, LWTFood Sci. Technol., 1995, 28, 25.

44. K. Mishra, H. Ojha, and N. K. Chaudhury, Food Chem., 2012, 130, 1036.

45. M. C. Foti, J. Agric. Food. Chem., 2015, 63, 8765.

46. J. L. S. Barrita and M. d. S. S. Sánchez, in "Oxidative Stress and Chronic Degenerative Diseases-A Role for Antioxidants", 2013, InTech.

47. R. Apak, M. Özyürek, K. Güçlü, and E. Çapanoğlu, J. Agric. Food Chem., 2016, 64, 1028.

48. M. Kosar, H. Dorman, O. Bachmayer, K. Baser, and R. Hiltunen, Chem. Nat. Compd., 2003, 39, 161.

49. B. Pratumvinit, N. Charoenkoop, S. Niwattisaiwong, G. J. Kost, and P. Tientadakul, Journal of Diabetes Science and Technology, 2016, 10, 1094. 